\title{
ERRATUM
}

Fragkiska Megaloudi

\section{Burnt sacrificial plant offerings in Hellenistic times: an archaeobotanical case study from Messene, Peloponnese, Greece}

Published online: 10 November 2006

(C) Springer-Verlag 2006

Veget Hist Archaeobot (2005) 14:329-340

Unfortunately there were some errors in Tables 3-4.

These tables are given correctly below.

The online version of the original article can be found at http://dx.doi.org/10.1007/s00334-005-0083-x

F. Megaloudi $(\square)$

Centre d'Anthropologie,

CNRS UMR 8555, 39 Allées Jules Guesde,

31000 Toulouse, France

e-mail: fmegaloudi@yahoo.fr

Present address:

F. Megaloudi

Department of Mediterranean Studies, University of the Aegean,

1 Dimokratias Av.,

85100 Rhodes, Greece 
Table 3 Other sites with remains of the species found in Messene. Key: PB = Public building, FC $=$ Funeral context, $\mathrm{SC}=$ Sacrificial context, $\mathrm{RM}=$ ritual meal

\begin{tabular}{|c|c|c|c|c|c|c|c|c|}
\hline Species & $\begin{array}{l}\text { Con- } \\
\text { text }\end{array}$ & $\begin{array}{l}\text { Pinus } \\
\text { pinea } \\
\text { nuts }\end{array}$ & $\begin{array}{l}\text { Pinus } \\
\text { pinea } \\
\text { bracts }\end{array}$ & $\begin{array}{l}\text { Pinus } \\
\text { pinea } \\
\text { cones }\end{array}$ & $\begin{array}{l}\text { Castanea } \\
\text { sativa }\end{array}$ & $\begin{array}{l}\text { Amyg- } \\
\text { dalus } \\
\text { communis }\end{array}$ & $\begin{array}{l}\text { Olea } \\
\text { europaea }\end{array}$ & $\begin{array}{l}\text { Vitis } \\
\text { vinifera }\end{array}$ \\
\hline $\begin{array}{l}\text { Doiikitirion, Thessalonica, Greece, } \\
\text { 1st cent. B.C.-end 2nd cent. A.D. (Mangaffa } \\
1998\end{array}$ & $\mathrm{~PB}$ & $\mathbf{x}$ & & & & & & \\
\hline $\begin{array}{l}\text { Watling Street, London, UK, } \\
\text { end 3rd cent. A.D. (Giorgi 2000) }\end{array}$ & $\mathrm{FC}$ & $\mathbf{X}$ & & & & & & \\
\hline $\begin{array}{l}\text { Temple of Mithras, London, UK, 1st/2nd } \\
\text { cent. A.D. (Kislev 1988) }\end{array}$ & $\mathrm{SC}$ & $\mathbf{x}$ & & & & & & \\
\hline $\begin{array}{l}\text { Staffordshire, UK, late 1st-mid/ } \\
\text { 2nd cent. A.D., (Monckton 2000) }\end{array}$ & $\mathrm{SC}$ & $\mathbf{x}$ & $\mathbf{x}$ & & & & & \\
\hline $\begin{array}{l}\text { Temple of Isis, Andalusia, Spain, } \\
\text { 2nd-3rd cent. A.D. (Lignereux et al. 1997) }\end{array}$ & $\mathrm{SC}$ & $\mathbf{X}$ & & & & & & \\
\hline $\begin{array}{l}\text { Isis and Magna Mater, Mainz, Germany, } \\
\text { 1st-4th cent. A.D. (Zach 2002) }\end{array}$ & $\mathrm{SC}$ & $\mathbf{X}$ & $\mathbf{X}$ & $\mathbf{X}$ & & & $\mathbf{X}$ & $\mathbf{x}$ \\
\hline $\begin{array}{l}\text { Salamine, Cyprus, } \\
\text { 4th cent. B.C., (Hjelmquist 1973) }\end{array}$ & $\mathrm{FC}$ & & & $\mathbf{x}$ & & & & \\
\hline $\begin{array}{l}\text { Tipasa, Algeria, } \\
\text { 1st-2nd cent. A.D. (Lancel 1970) }\end{array}$ & $\mathrm{FC}$ & & & $\mathbf{x}$ & & & & \\
\hline $\begin{array}{l}\text { Necropolis of Valladas Drome, France, } \\
\text { 2nd cent. A.D. (Marinval 1993) }\end{array}$ & $\mathrm{FC}$ & & & $\mathbf{X}$ & & & & \\
\hline $\begin{array}{l}\text { Necropolis Saint Lambert (Var), France, } \\
\text { 2nd cent. A.D. (Marinval 1993) }\end{array}$ & $\mathrm{FC}$ & & & $\mathbf{x}$ & & & & \\
\hline $\begin{array}{l}\text { Pontarion (Creuse), France, } \\
\text { end 3rd cent. A.D. (Marinval 1993) }\end{array}$ & $\mathrm{FC}$ & & & $\mathbf{x}$ & & & & \\
\hline $\begin{array}{l}\text { Cova des Carritx, Spain, } \\
\text { Late Bronze Age (Stika 1999) }\end{array}$ & $?$ & & & & $\mathbf{x}$ & & & \\
\hline $\begin{array}{l}\text { Crimea, Ukraine, } \\
\text { 4th cent. B.C. (Wasylikowa et al. 1991) }\end{array}$ & $\mathrm{FC}$ & & & & $\mathbf{x}$ & & & \\
\hline $\begin{array}{l}\text { Pompeii, Oplontis and Herculaneum, Italy, } \\
\text { 1st cent. A.D. (Overbeck and Mau 1884) }\end{array}$ & $\mathrm{SC}$ & & & & $\mathbf{x}$ & & & \\
\hline $\begin{array}{l}\text { Heraion Samos, Greece, } \\
\text { 7th cent. B.C. (Kucan 1995) }\end{array}$ & $\mathrm{SC}$ & & & & & $\mathbf{x}$ & $\mathbf{X}$ & $\mathbf{x}$ \\
\hline $\begin{array}{l}\text { Sanctuary of Demeter and Core, Corinth, } \\
\text { Greece, 5th cent. B.C. (Bookidis et al. 1999) }\end{array}$ & $\mathrm{SC}$ & & & & & & $\mathbf{x}$ & \\
\hline $\begin{array}{l}\text { "Pilier des Rhodies", Delphi, Greece, } \\
\text { 8th-7th cent. B.C. (Luce et al. in press) }\end{array}$ & RM & & & & & & $\mathbf{x}$ & $\mathbf{x}$ \\
\hline $\begin{array}{l}\text { Sanctuary of Kalapodi, Greece, } \\
\text { 10th-9th cent. B.C. (Kroll 1993) }\end{array}$ & RM & & & & & & $\mathbf{x}$ & $\mathbf{x}$ \\
\hline $\begin{array}{l}\text { Roman Palaimonion, Greece, Pit A, } \\
\text { 50-100 A.D. (Gebhard 1993) }\end{array}$ & $\mathrm{SC}$ & & $\mathbf{X}$ & & & & & \\
\hline $\begin{array}{l}\text { Palaimonion, Greece, Pit C, late 2nd-early } \\
\text { 3rd cent. B.C. (cited in Bookidis et al. 1999) }\end{array}$ & $\mathrm{SC}$ & & $\mathbf{x}$ & & & & & \\
\hline $\begin{array}{l}\text { La Favorite (F), Valkenburg (NL), } \\
\text { Vindonissa }(\mathrm{CH}), 1 \text { st-2nd A.D. (compiled } \\
\text { by Petrucci-Bavaud and Jacomet } 1997\end{array}$ & $\mathrm{FC}$ & & & & & & & $\mathbf{x}$ \\
\hline $\begin{array}{l}\text { Kempten (D), 1st cent. A.D. (compiled by } \\
\text { Petrucci-Bavaud and Jacomet } 1997\end{array}$ & $\mathrm{FC}$ & & & & $\mathbf{x}$ & & & \\
\hline
\end{tabular}



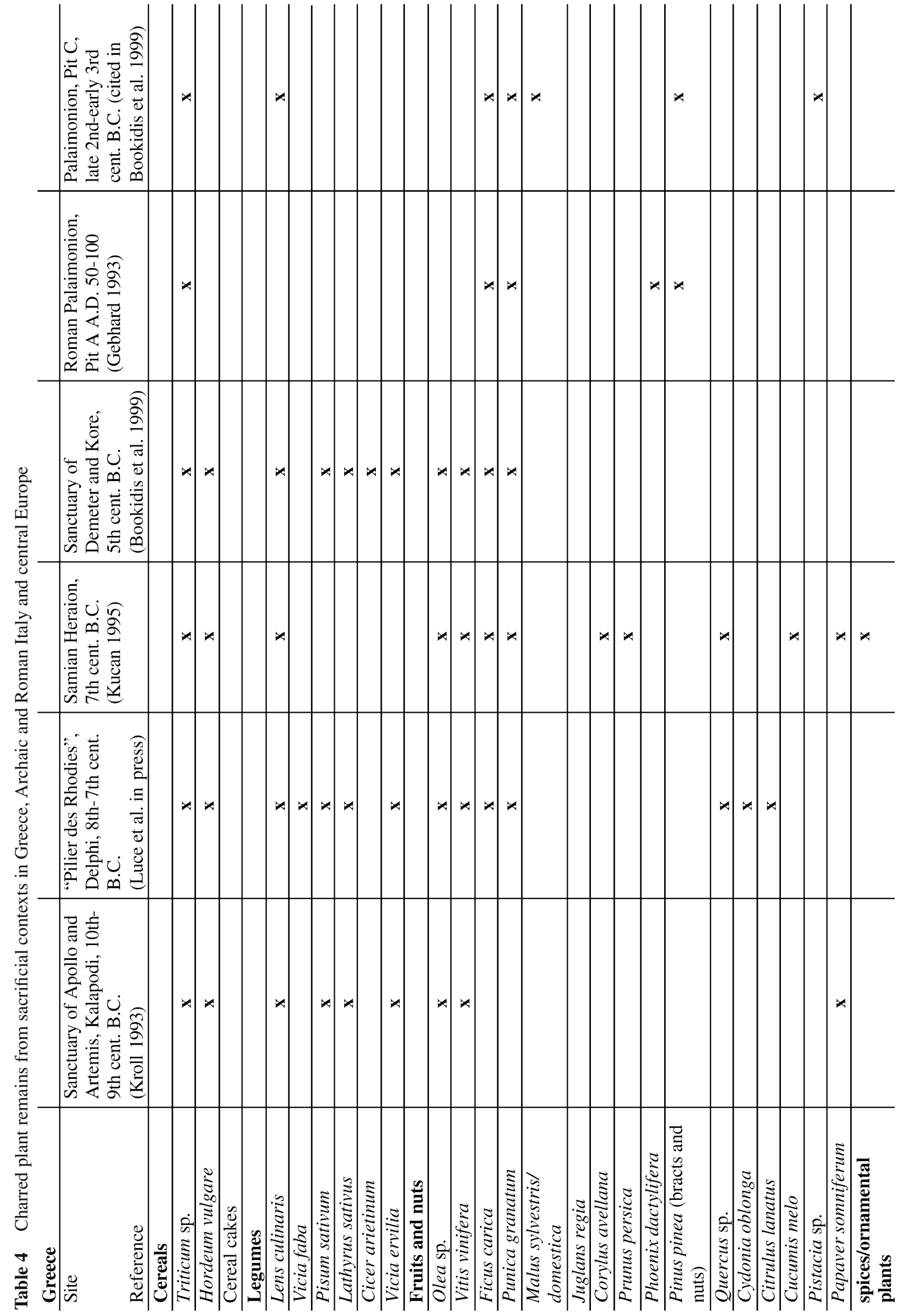

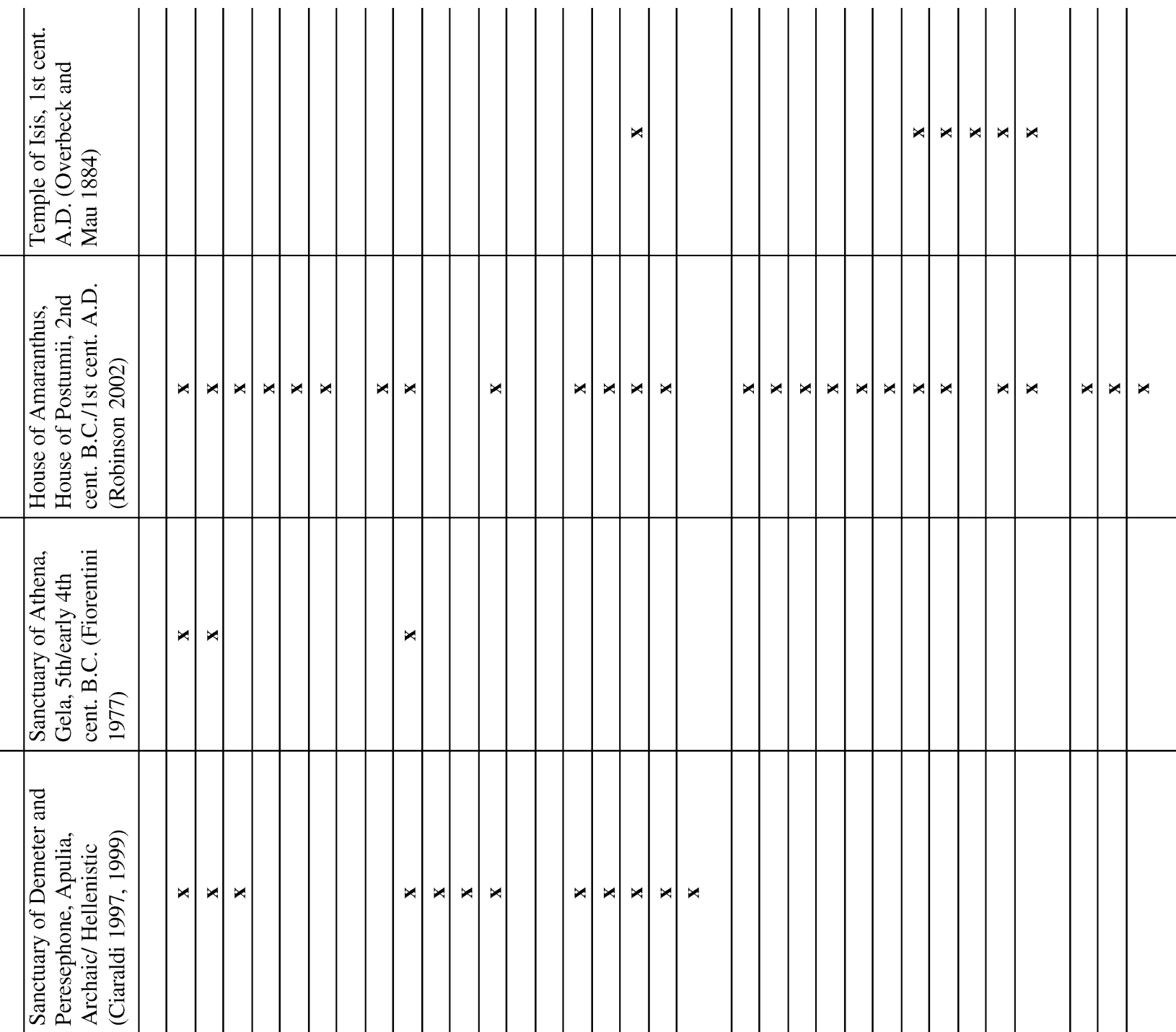

흘.

可

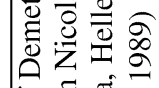

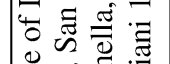



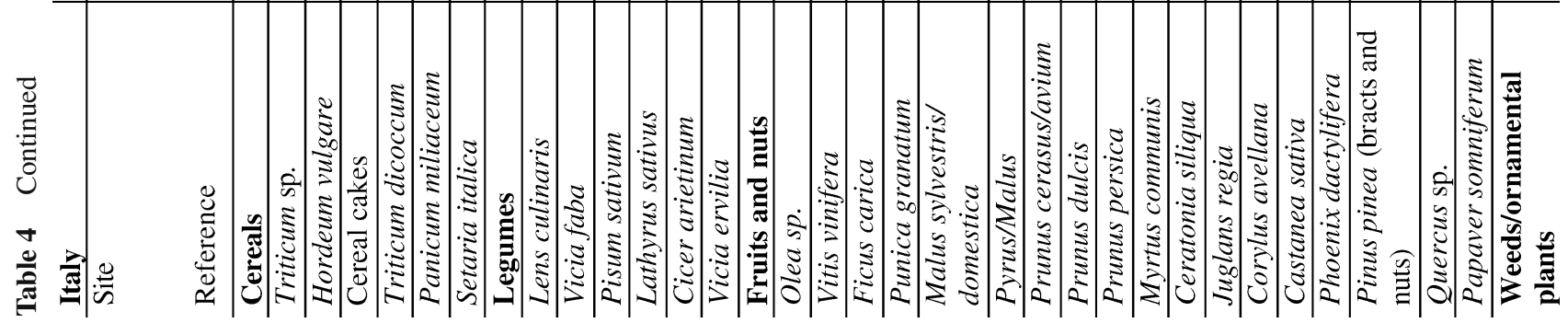



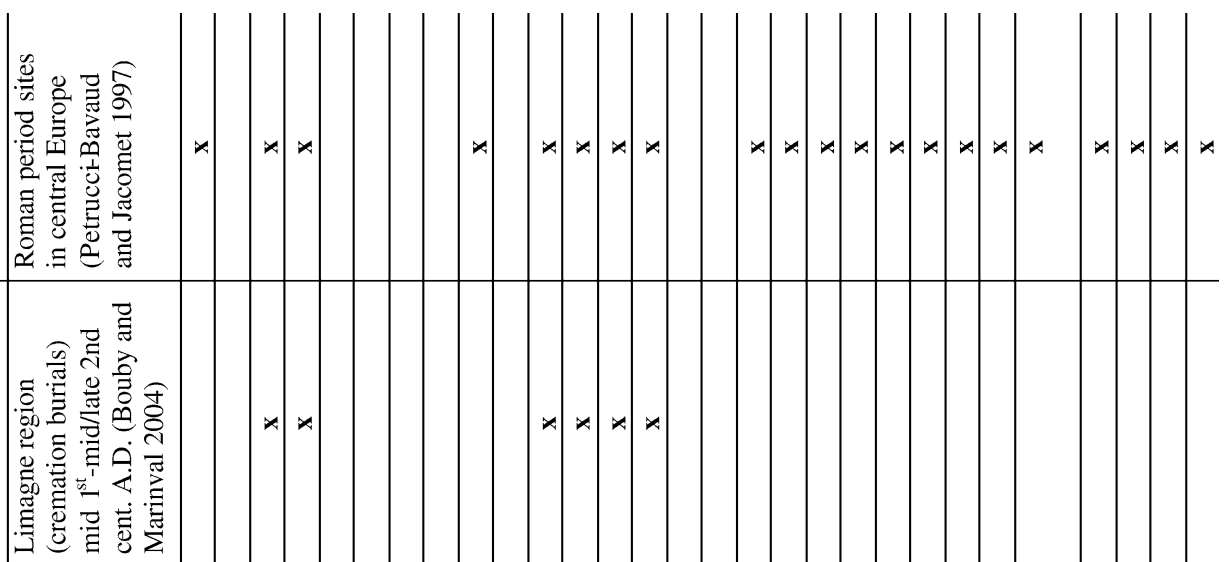

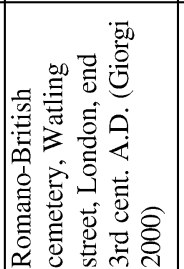

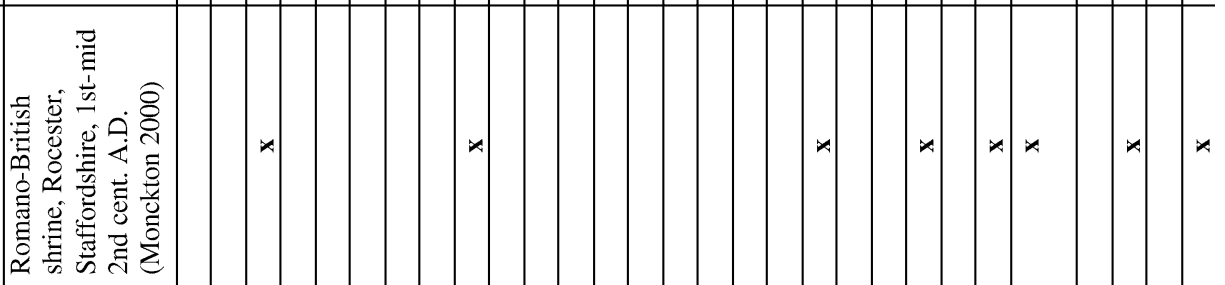


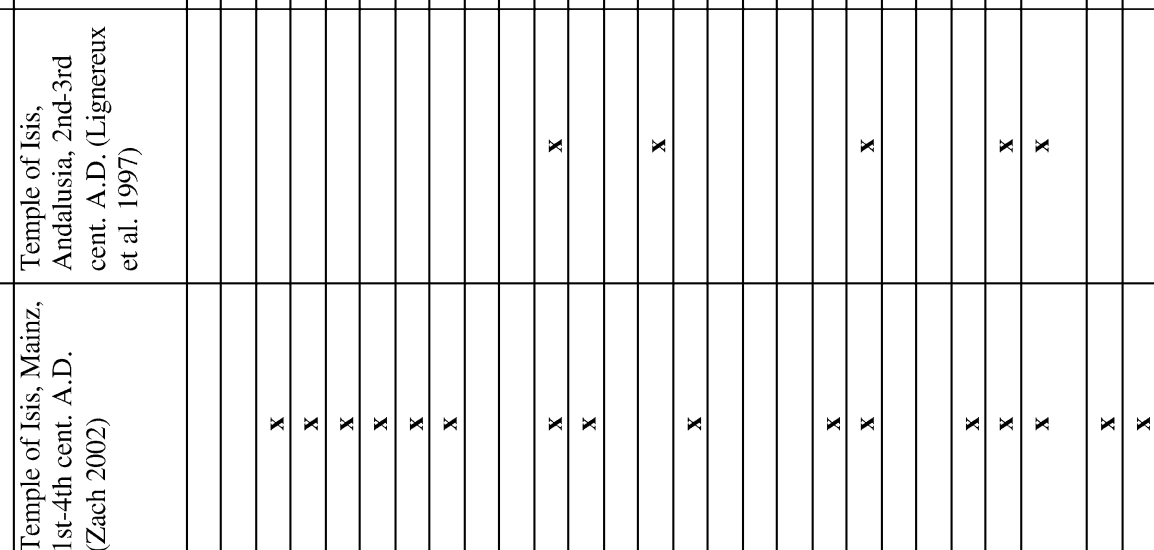

意点, 